\title{
Shaping Biphoton Temporal Waveforms with Modulated Classical Fields
}

\author{
J. F. Chen, Shanchao Zhang, Hui Yan, M. M. T. Loy, G. K. L. Wong, and Shengwang Du* \\ Department of Physics, The Hong Kong University of Science and Technology, Clear Water Bay, Kowloon, Hong Kong, China
} (Received 3 February 2010; published 6 May 2010)

\begin{abstract}
We experimentally demonstrate a technique for shaping the temporal quantum waveform of narrowband biphotons generated in a cold atomic ensemble via four-wave mixing by periodically modulating the two input classical lasers. We show that it is possible to generate nonclassical paired photons with a predesigned shape of the correlation function.
\end{abstract}

DOI: 10.1103/PhysRevLett.104.183604

PACS numbers: 42.50.Dv, 32.80.Qk, 42.50.Gy, 42.65.Lm

Entangled paired photons, termed biphotons, are at the heart of the research field of quantum optics. Generating single photons with controllable quantum states is of particular interest to quantum communication [1], quantum information processing [2], quantum computation [3], quantum imaging [4], quantum lithography [5], and quantum metrology [6]. The biphotons are characterized by their intrinsic spins (polarizations) and time-space wave functions. While manipulation of single- and multiplephoton polarization states has found important applications in quantum communication [1], access to biphotons with controllable time-space waveforms is still limited. For biphotons produced from conventional spontaneous parametric down-conversion (SPDC) $[7,8]$, this difficulty is due mainly to their wide bandwidth $(\sim \mathrm{THz})$, short coherence time $(\sim \mathrm{ps})$, as well as to the time resolution $(\sim \mathrm{ns})$ of stateof-the-art single-photon counting modules (SPCM). As a result, shaping SPDC photon temporal waveforms can be achieved only by performing spectral manipulation of the joint spectrum [9-11] or spatial modulation of the nonlinear interaction [12-14]. Nonlocal modulation of entangled photons has also been observed $[15,16]$. The temporal length of these photons can be lengthened by putting the nonlinear crystal inside a cavity [17-19]. However, the waveforms are determined by the cavity modes and hence not tailorable.

Recent demonstration of generation of narrow-band biphotons in atomic ensembles with long coherence time $(0.1-1 \mu \mathrm{s})$ via four-wave mixing (FWM) made it possible to measure their time-domain quantum waveforms directly using SPCMs [20-22]. By passing these long single photons through electro-optical modulators, it is possible to modulate one of the paired photons [23] or their correlation function [24]. However, these external modulators always introduce losses and attenuations to the single photons. Moreover, in these experiments $[23,24]$ where amplitude modulation is performed using Mach-Zehnder interferometers, coupling between the single photons and the environment at the beam splitters introduces noises (uncorrelated photons) to the system.

In this Letter, we report experimental demonstration of shaping the temporal quantum waveform of narrow-band biphotons by periodically modulating the two driving fields in the FWM process, which was first predicted by Du et al. [25]. We show the input field profiles can be revealed in the two-photon waveform. We also demonstrate the generation of nonclassical paired photons with arbitrarily shaped correlation function. Our result can be considered as the temporal analog of the spatial modulation technique introduced by Monken $e t$ al. by which the angular spectrum of the pump beam can be transferred to the biphoton transverse correlation [26].

The schematics of narrow-band biphoton generation is shown in Fig. 1. We work with a two-dimensional (2D) ${ }^{85} \mathrm{Rb}$ magneto-optical trap (MOT) with a longitudinal length $L=1.7 \mathrm{~cm}$ and a temperature of about $100 \mu \mathrm{K}$. The atoms are prepared in the ground state $|1\rangle$. In the presence of counterpropagating, circularly polarized, and periodically modulated pump $\left(\omega_{p}\right)$ and coupling $\left(\omega_{c}\right)$ lasers, phase matched Stokes $\left(\omega_{s}\right)$ and anti-Stokes $\left(\omega_{\text {as }}\right)$ photons are spontaneously generated in opposite directions. After passing through a set of polarization selectors (consisting of a quarter-wave plate and a polarization beam splitter) and narrow-band filters $\left(F_{1}\right.$ and $F_{2}, 0.5 \mathrm{GHz}$ bandwidth), these paired photons are coupled into opposing single-mode fibers (SMF) and detected by SPCMs (Perkin Elmer SPCM-AQ4C). Both lasers are transmitted through $10 \mathrm{GHz}$ electro-optical (EO) amplitude modulators (EOspace, Inc.), whose waveforms are generated using a function generator (Tektronix AFG3252) with two output

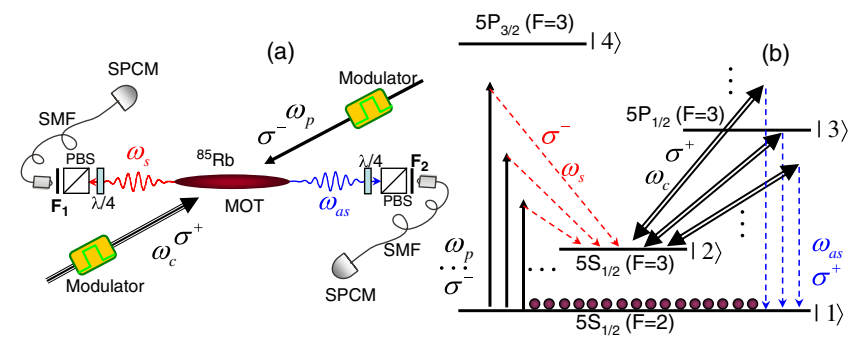

FIG. 1 (color online). Schematic of biphoton generation with modulated driving-laser fields. (a) Experimental configuration. (b) ${ }^{85} \mathrm{Rb}$ double- $\Lambda$ energy level diagram for multichannel biphoton generation. 
channels. The counterpropagating pump and coupling beams are aligned at $2^{\circ}$ angle from the Stokes-antiStokes direction. As shown in Fig. 1(b), the periodically modulated pump and coupling laser fields are decomposed in frequency domain into discrete frequency components. As a result, biphoton generation follows many possible FWM paths. The interference between these multichannel FWMs provides a controllable way to manipulate and engineer the biphoton wave packets.

In our work, the pump laser is far detuned from the $|1\rangle \rightarrow|4\rangle$ transition $\left(\Delta_{p}=2 \pi \times 146 \mathrm{MHz}\right)$ so that the Stokes photons travel at nearly the speed of light in vacuum with negligible Raman gain. In contrast, the unmodulated coupling laser is on resonance with the transition $|2\rangle \rightarrow|3\rangle$, and it renders electromagnetically induced transparency (EIT) [27] to the anti-Stokes photons that travel at a slower group velocity. As the coupling laser is modulated periodically, the spontaneously emitted anti-Stokes photons automatically match their coupling discrete frequency components. While biphoton generation in the double- $\Lambda$ atomic system with continuous-wave (cw) pump and coupling lasers is well understood [28], the near-atomicresonance nonlinear process with time-varying pump and coupling lasers has not been solved analytically. However, when the atomic optical depth in the transition $|1\rangle \rightarrow|3\rangle$ is high and the EIT slow-light effect is significant, the theoretical analysis becomes greatly simplified. Under these conditions, following the theory by $\mathrm{Du}$ et al. [25], we obtain the biphoton wave function in the Schrödinger picture

$$
\Psi\left(t_{s}, t_{\mathrm{as}}\right)=\frac{c \varepsilon_{0}}{2 \sqrt{\bar{I}_{p} \bar{I}_{c}}} E_{p}\left(t_{s}\right) E_{c}\left(t_{\mathrm{as}}\right) \psi_{0}\left(t_{\mathrm{as}}-t_{s}\right) e^{-i \omega_{s 0} t_{s}} e^{-i \omega_{\mathrm{as} 0} t_{\mathrm{as}}},
$$

where $E_{p}\left(t_{s}\right)$ and $E_{c}\left(t_{\mathrm{as}}\right)$ are the complex envelopes of the pump and coupling laser fields. $\bar{I}_{p}$ and $\bar{I}_{c}$ are the pump and coupling laser average intensities. $\omega_{s 0}$ and $\omega_{\text {as } 0}$ are Stokes and anti-Stokes central angular frequencies. $\psi_{0}\left(t_{\mathrm{as}}-\right.$ $\left.t_{s}\right) e^{-i \omega_{s 0} t_{s}} e^{-i \omega_{\text {as } 0} t_{\text {as }}}$ is the original two-photon wave packet without modulation on the driving fields. As shown in Eq. (1), the two-photon time-frequency entanglement information is preserved while the pump and coupling field profiles are mapped into the biphoton waveform. The Stokes and anti-Stokes temporal correlation function is

$$
R\left(t_{s}, t_{\mathrm{as}}\right) \equiv\left|\Psi\left(t_{s}, t_{\mathrm{as}}\right)\right|^{2}=\frac{I_{p}\left(t_{s}\right) I_{c}\left(t_{\mathrm{as}}\right)}{\bar{I}_{p} \bar{I}_{c}} R_{0}(\tau),
$$

where $\tau=t_{\text {as }}-t_{s} . I_{p}\left(t_{s}\right)$ and $I_{c}\left(t_{\mathrm{as}}\right)$ are the pump and coupling laser intensity temporal profiles. $R_{0}(\tau)=$ $\left|\psi_{0}(\tau)\right|^{2}$ is the correlation function without modulation. Therefore the time-averaged correlation becomes

$$
\bar{R}(\tau)=C(\tau) R_{0}(\tau)
$$

where $C(\tau) \equiv \lim _{\Delta T \rightarrow \infty} \frac{1}{\Delta T \bar{I}_{p} \bar{I}_{c}} \int_{0}^{\Delta T} I_{p}(t) I_{c}(t+\tau) d t$ is the time-averaged pump-coupling correlation function. Equations (1)-(3) show that it is possible to manipulate the biphoton temporal wave packet and its correlation functions in a controllable way.

We first determine the two-photon correlation with $\mathrm{cw}$ driving lasers. The experiment is run periodically with $4.5 \mathrm{~ms}$ MOT trapping time and $0.5 \mathrm{~ms}$ biphoton generation window. The optical depth at the anti-Stokes transition is about 35 . The unmodulated pump and coupling lasers have Rabi frequencies of $\Omega_{p 0}=1.0 \gamma_{13}$ and $\Omega_{c 0}=3.0 \gamma_{13}$, where $\gamma_{13}=2 \pi \times 3 \mathrm{MHz}$ is the dipole relaxation rate between $|1\rangle$ and $|3\rangle$. The measured ground state dephasing rate between $|1\rangle$ and $|2\rangle$ is $\gamma_{12}=0.01 \gamma_{13}$. The two-photon coincidence counts per $1 \mathrm{~ns}$ bin for $120 \mathrm{~s}$ run time is shown in the Fig. 2, plot (1). There are several features in the twophoton correlation function. The long temporal correlation time of about $500 \mathrm{~ns}$ is consistent with the slow-light group delay of the anti-Stokes photons [22]. The oscillatory structure on top of the leading edge is optical precursor at the biphoton level [29]. Taking into account the fiberfiber coupling efficiency (70\%), filter transmission (80\%), detector quantum efficiency $(50 \%)$, and the duty cycle $(10 \%)$, the system spontaneously generates 80000 pair photons per second. The theoretical curve (solid line), calculated using the Schrödinger picture approach [28], agrees well with the experimental data. With the Stokes and anti-Stokes single-photon rates $R_{s}$ and $R_{\text {as }}$, we determine the normalized cross-correlation function $g_{s, \text { as }}^{(2)}(\tau)=$ $\bar{R}(\tau) /\left(R_{S} R_{\mathrm{as}}\right)$ that peaks at a value of 10 . We also measure the autocorrelations of the Stokes and anti-Stokes photons using a fiber beam splitter, and obtain $g_{s, s}^{(2)}(0) \simeq g_{\mathrm{as}, \mathrm{as}}^{(2)}(0) \simeq$ 2.0. We then obtain violation of the Cauchy-Schwartz inequality $\left[g_{s, \text { as }}^{(2)}(\tau)\right]^{2} /\left[g_{s, s}^{(2)}(0) g_{\text {as, as }}^{(2)}(0)\right] \leq 1[30]$ by a factor of more than 25 and thus verify the nonclassical nature of the entangled photons.

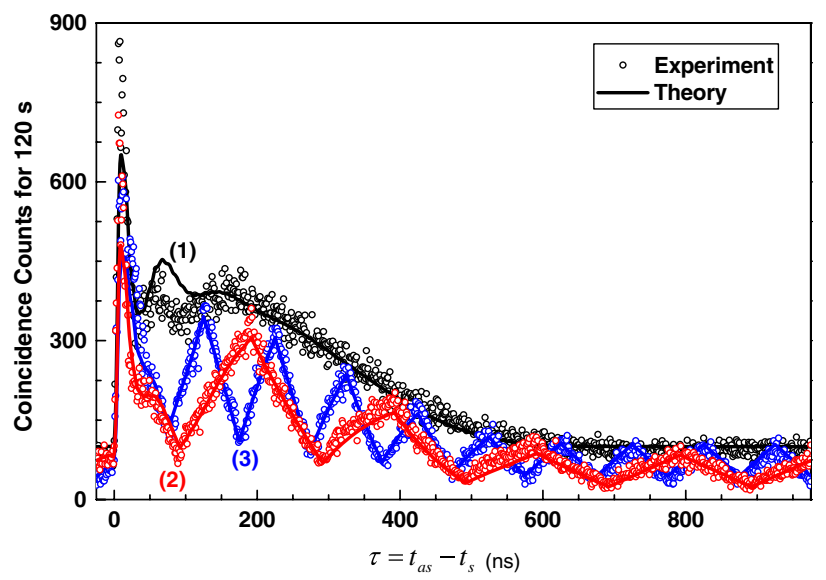

FIG. 2 (color online). Two-photon coincidence counts per $1 \mathrm{~ns}$ bin for $120 \mathrm{~s}$ as a function of the relative time delay $\tau$. In (1), The pump and coupling lasers are in the cw mode. In (2) and (3), both pump and coupling lasers are periodically modulated with the same square wave at a frequency of 5 and $10 \mathrm{MHz}$, respectively. 
We now apply identical square amplitude modulation to both pump and coupling lasers. In order to make both qualitative and quantitative comparisons with the unmodulated waveform, we maintain the same average coupling laser intensity, i.e., $\bar{I}_{c}=I_{c 0}$, where $I_{c 0}$ is the cw coupling laser intensity, because the anti-Stokes group delay and thus the two-photon coherence time is determined by the average intensity of the coupling laser [25]. The peak intensity of the pump laser remains the same. Plots (2) and (3) in Fig. 2 show the measured two-photon correlations with a modulation frequency of 5 and $10 \mathrm{MHz}$, respectively. As expected from Eq. (3), the intensity correlation of triangle shapes between the pump and coupling lasers are transferred into the biphoton time-averaged correlation function. The theoretical predictions (solid lines) by Eqs. (1)-(3) are in excellent agreement with the experimental data. We have also measured the autocorrelation functions and verify that the violation of the CauchySchwartz inequality is not degraded by the modulation.

To reveal the full information encoded by the modulated pump and coupling lasers, as described in Eqs. (1) and (2), we measure the 2D Stokes and anti-Stokes temporal correlation with $10 \mathrm{MHz}$ square modulation on both lasers. As displayed in Fig. 3, both the unmodulated biphoton correlation envelope $R_{0}(\tau)$ and square modulations $\left|E_{p}\left(t_{s}\right) E_{c}\left(t_{\mathrm{as}}\right)\right|^{2}$ are shown in the two-photon nonclassical correlation. The straight line along the $45^{\circ}$ in Fig. 3(a) is the leading edge of the precursor [29]. The asymmetry of the distribution is due to the EIT slow-light effect that delays the anti-Stokes photons while the Stokes travel at nearly the speed of light in vacuum $[22,28]$. As a result, the anti-Stokes photon is always generated later than the paired Stokes $\left(t_{\text {as }}>t_{s}\right)$. We note that below the $45^{\circ}$ line $\left(t_{\mathrm{as}}<t_{s}\right)$, there are still square modulations on the background floor with flat tops [Fig. 3(d)]. This modulation is on the accidental coincidence between the uncorrelated Stokes and anti-Stokes photons, or a simple product of their single-photon counting rates. During the dark periods there are barely any coincidence counts, because the single counts disappear as one or both driving fields are turned off. We further confirm that within the biphoton correlation time [Figs. 3(b) and 3(c)] the Cauchy-Schwartz inequality is violated without degradation, and the inequality is not violated in the noncorrelated regime [Fig. 3(d)].

Figure 4 shows the result of time-averaged correlation function with both lasers modulated by a $5 \mathrm{MHz}$ sine waveform. In such a case, the input pump-coupling intensity correlation is $C(\tau) \propto\left[2+\cos \left(2 \omega_{m} \tau\right)\right] / 8$, that is revealed also in the biphoton correlation.

In the above cases, we have shown experimental results with the two driving classical laser fields modulated by the same periodical waveform. However, the theoretical prediction of Eq. (1) is not limited to identical modulations. When the pump and coupling lasers are modulated by different waveforms, it is possible to generate two-photon correlation with arbitrary waveforms. To verify this, we

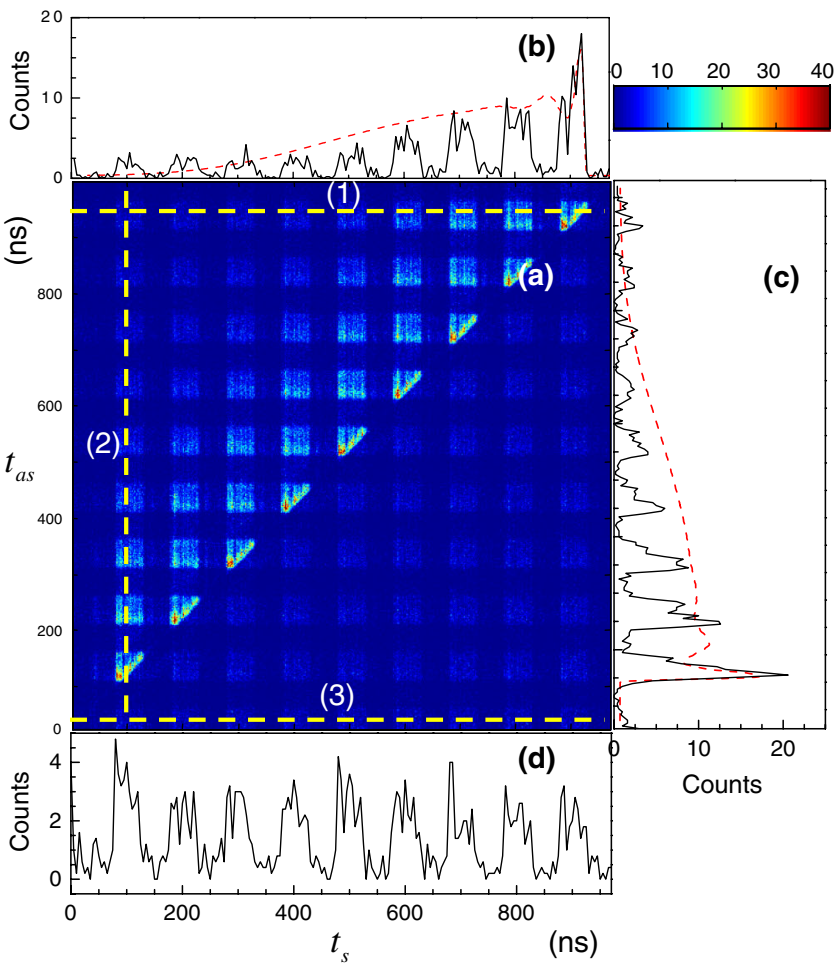

FIG. 3 (color online). (a) The 2D two-photon correlation function $R\left(t_{s}, t_{\mathrm{as}}\right)$ with $10 \mathrm{MHz}$ square amplitude modulation on both pump and coupling lasers. (b), (c), and (d) are cross-section views (averaged over 5 bins) along the yellow dashed straight lines (1), (2), and (3) in (a), respectively. The red dashed lines in (b) and (c) are the correlation envelope $R_{0}\left(t_{\mathrm{as}}-t_{s}\right)$ without modulation. The data were collected over 20 min with 1 ns bin width.

modulate the two lasers with different waveforms. As an example, we modulate the pump laser by short Gaussian pulses with a width of $10 \mathrm{~ns}$ and a repetition rate of $1 \mathrm{MHz}$. The coupling laser is modulated by a square followed by a

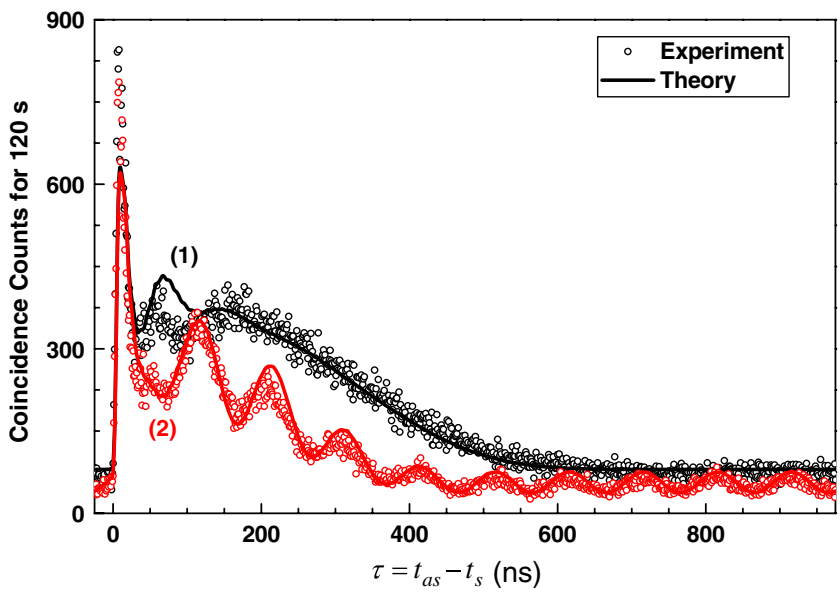

FIG. 4 (color online). Two-photon coincidence counts per $1 \mathrm{~ns}$ bin for $120 \mathrm{~s}$ as a function of the relative time delay $\tau$. In (1), The pump and coupling lasers are in the cw mode. In (2) both pump and coupling lasers are modulated with the same sine wave at a frequency of $5 \mathrm{MHz}$. 


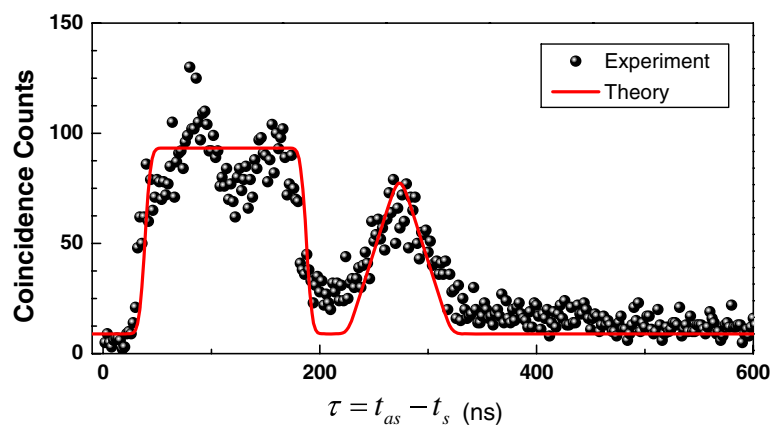

FIG. 5 (color online). The proof-of-principle demonstration for generating arbitrary two-photon correlation function with nonidentical modulations on the pump and coupling lasers. The solid line is the targeted waveform. The data (circular points) was collected over 10 min with 2 ns bin width.

triangle shape with the same modulation period. In this way, we expect to generate a correlation function with the shape of the modulation waveform imposed on the coupling laser. To do so, we have compensated the nonlinear transfer function of the EO amplitude modulators. The measured two-photon correlation is shown in Fig. 5, in good agreement with the theoretical prediction. Therefore, we have demonstrated a proof-of-principle experimental realization of generation of biphotons having a temporal correlation function whose shape can be arbitrarily controlled. Our result may find potential application in quantum communication and quantum information science.

It is clear that in order to obtain the full modulation information on the biphoton waveform, it is necessary to measure the 2D temporal correlation function $R\left(t_{s}, t_{\mathrm{as}}\right)$, as shown in Fig. 3. However, this requires long measurement time. On the other side, one can measure the time-averaged correlation functions in a much shorter time, which, together with the violation of the Cauchy-Schwartz inequality, provide (though not the full) information about the nonclassical properties of the biphotons. Moreover, the quantum coincidence is linearly proportional to the single-photon rate, while the classical coincidence scales quadratically to the single-photon rate.

Finally, we should mention that the technique described in this Letter cannot be fully mimicked in the conventional SPDC, where modulation on the single pump beam has an impact on the nonclassical correlation and entanglement [31], since it affects the biphoton waveform only in the direction $t_{s}+t_{i}$ but not $t_{i}-t_{s}$, where $t_{s}$ and $t_{i}$ are the time variables for the signal and idler photons.
The work was supported by the Hong Kong Research Grants Council (Project No. HKUST600809). Some equipment used in the experiment was provided by the William Mong Clusters Laboratory and the Joyce M. Kuok Lasers and Photonics Laboratory.

*dusw@ust.hk

[1] N. Gisin et al., Rev. Mod. Phys. 74, 145 (2002).

[2] S. L. Braunstein and P. van Loock, Rev. Mod. Phys. 77, 513 (2005).

[3] M. Neilson and I. Chuang, Quantum Computation and Quantum Information (Cambridge University Press, Cambridge, U.K., 2000).

[4] M. D’Angelo and Y.-H. Shih, Laser Phys. Lett. 2, 567 (2005).

[5] M. D’Angelo, M. V. Chekhova, and Y. Shih, Phys. Rev. Lett. 87, 013602 (2001).

[6] A. Migdall et al., Appl. Opt. 37, 3455 (1998).

[7] S. E. Harris et al., Phys. Rev. Lett. 18, 732 (1967).

[8] D. Burnham and D. Weinberg, Phys. Rev. Lett. 25, 84 (1970).

[9] A. Pe'er et al., Phys. Rev. Lett. 94, 073601 (2005).

[10] S. Viciani, A. Zavatta, and M. Bellini, Phys. Rev. A 69, 053801 (2004).

[11] M. Hendrych et al., Phys. Rev. A 79, 023817 (2009).

[12] A. Valencia et al., Phys. Rev. Lett. 99, 243601 (2007).

[13] S. E. Harris, Phys. Rev. Lett. 98, 063602 (2007).

[14] M. B. Nasr et al., Phys. Rev. Lett. 100, 183601 (2008).

[15] S. E. Harris, Phys. Rev. A 78, 021807(R) (2008).

[16] S. Sensarn, G. Y. Yin, and S. E. Harris, Phys. Rev. Lett. 103, 163601 (2009).

[17] Z. Y. Ou and Y. J. Lu, Phys. Rev. Lett. 83, 2556 (1999).

[18] C. E. Kuklewicz, F. N. C. Wong, and J. H. Shapiro, Phys. Rev. Lett. 97, 223601 (2006).

[19] X.-H. Bao et al., Phys. Rev. Lett. 101, 190501 (2008).

[20] V. Balić et al., Phys. Rev. Lett. 94, 183601 (2005).

[21] S. Du et al., Phys. Rev. Lett. 98, 053601 (2007).

[22] S. Du et al., Phys. Rev. Lett. 100, 183603 (2008).

[23] P. Kolchin et al., Phys. Rev. Lett. 101, 103601 (2008).

[24] C. Belthangady et al., Phys. Rev. A 80, 031803(R) (2009).

[25] S. Du, J. Wen, and C. Belthangady, Phys. Rev. A 79, 043811 (2009).

[26] C. H. Monken, P. H. Souto Ribeiro, and S. Pádua, Phys. Rev. A 57, 3123 (1998).

[27] S. E. Harris, Phys. Today 50, No. 7, 36 (1997).

[28] S. Du, J. Wen, and M. H. Rubin, J. Opt. Soc. Am. B 25, C98 (2008).

[29] S. Du et al., Opt. Lett. 33, 2149 (2008).

[30] J. F. Clauser, Phys. Rev. D 9, 853 (1974).

[31] T.E. Keller and M.H. Rubin, Phys. Rev. A 56, 1534 (1997). 